University of Nebraska - Lincoln

DigitalCommons@University of Nebraska - Lincoln

January 2006

\title{
The elastic constants and related properties of the energetic material cyclotrimethylene trinitramine (RDX) determined by Brillouin scattering
}

\author{
James J. Haycraft \\ University of Nebraska - Lincoln \\ Lewis L. Stevens \\ University of Nebraska - Lincoln \\ Craig J. Eckhardt \\ University of Nebraska - Lincoln, ceckhardt1@unl.edu
}

Follow this and additional works at: https://digitalcommons.unl.edu/chemistryeckhardt

Part of the Chemistry Commons

\footnotetext{
Haycraft, James J.; Stevens, Lewis L.; and Eckhardt, Craig J., "The elastic constants and related properties of the energetic material cyclotrimethylene trinitramine (RDX) determined by Brillouin scattering" (2006). Craig J. Eckhardt Publications. 2.

https://digitalcommons.unl.edu/chemistryeckhardt/2
}

This Article is brought to you for free and open access by the Published Research - Department of Chemistry at DigitalCommons@University of Nebraska - Lincoln. It has been accepted for inclusion in Craig J. Eckhardt Publications by an authorized administrator of DigitalCommons@University of Nebraska - Lincoln. 


\title{
The elastic constants and related properties of the energetic material cyclotrimethylene trinitramine (RDX) determined by Brillouin scattering
}

\author{
James J. Haycraft, Lewis L. Stevens, and Craig J. Eckhardt ${ }^{\mathrm{a})}$ \\ Department of Chemistry and Center for Materials Research and Analysis, University of Nebraska-Lincoln, \\ Lincoln, Nebraska 68588-0304
}

(Received 24 June 2005; accepted 3 November 2005; published online 10 January 2006)

\begin{abstract}
The acoustic phonons of cyclotrimethylene trinitramine (RDX) have been studied using Brillouin scattering. The analysis of the acoustic-phonon velocities allowed determination of the complete stiffness tensor for this energetic material. The results are compared to other recent experimental and theoretical determinations of the RDX elastic constants, bulk moduli, and shear moduli. The observed ordering of elastic constants, $C_{11}>C_{22}>C_{33}$, is qualitatively associated with a (001) cleavage plane and molecular packing. This interpretation is further corroborated by the linear compressibilities plotted in three crystallographic planes, and a comparison to recent theoretical and experimental hydrostatic compression studies on RDX. Finally, the elasticity of RDX is compared to a recently published report on the beta polymorph of cyclotetramethylene tetranitramine's elasticity, and is related to several proposed mechanisms for detonation initiation. (C) 2006 American Institute of Physics. [DOI: 10.1063/1.2141958]
\end{abstract}

\section{INTRODUCTION}

Organic solid-state chemistry explores the chemical reactions and intermolecular interactions of molecular crystals. Starting with the pioneering work of Schmidt ${ }^{1}$ and his topochemical postulate, ${ }^{2}$ this field has expanded to include crystal engineering and mechanochemistry. Interest has recently been rekindled in the microscopic behavior of a particularly interesting class of solid-state chemical reactionsthose that undergo detonation. While it is fairly simple to identify whether something has detonated, the actual process by which this phenomenon is initiated on the molecular level remains a matter of debate. As a consequence, one of the oldest solid-state chemical processes known is also one of the least understood, and this fact has led to renewed interest in ascertaining the microscopic mechanism that initiates detonation.

Detonation can be considered to be a collective property of the material and is highly dependent upon intermolecular interactions, molecular arrangements, and molecular composition. The strength of the interactions between the molecules comprising a molecular crystal has a measurable effect on the macroscopic properties of the solid. It is known that the various polymorphs of cyclotetramethylene tetranitramine (HMX) have different detonation properties, ${ }^{3}$ suggesting that different packings of the lattice have a significant effect on the material's response to impact. These properties can often be correlated to the strength of the lattice interactions through the elastic constants. An example is the energetic material pentaerithrytol tetranitrate (PETN), whose stiffest elastic constant ${ }^{4}$ is $C_{11}$, and lowest detonation sensitivity is along the $a$ crystallographic axis. ${ }^{5}$ The mechanisms that make this behavior possible have not been fully investigated.

\footnotetext{
${ }^{a)}$ Author to whom correspondence should be addressed. Electronic mail: eckhardt@unlserve.unl.edu
}

The importance of the lattice in the initiation of detonation in solid energetic materials cannot be understated. It is the lattice interactions that are of primary interest because they hold the answer to how energy (mechanical, thermal, or electrical) is transferred not only from one part of the solid to another but also from the lattice to the molecules. This transfer of energy is necessary for the initiation of detonation that then leads to the subsequent decomposition reaction. To gain an understanding of the mechanism of detonation initiation, a series of studies on mechanical properties needs to be performed on several energetic materials. Recently, our laboratory published a comprehensive study on the elastic properties of the energetic material $\beta$-HMX at ambient conditions. ${ }^{6}$ Cyclotrimethylene trinitramine (RDX) is a typical secondary explosive, as is $\beta$-HMX, and therefore is a logical choice for the determination of elastic properties, for comparison to those of other energetic materials, and for understanding how those properties relate to detonation. There have been several previous reports of the elastic constants of RDX. A resonant ultrasonic study reported by Haussühl ${ }^{7}$ was for single crystals of RDX at $293 \mathrm{~K}$ and ambient pressure. Another very recent resonant ultrasonic study by Schwarz et al. ${ }^{8}$ was for RDX at $298 \mathrm{~K}$ and ambient pressure conditions. A theoretical calculation of Ye et al. ${ }^{9}$ was for RDX at $298 \mathrm{~K}$ and ambient pressure. Ye et al. employed the software package of the general utility lattice program (GULP) ${ }^{10}$ using a fully flexible model of an energetic molecular crystal, originally developed by Sorescu et al. ${ }^{11}$ for nitromethane. Using potentials and parameters from Thompson and co-workers ${ }^{12,13}$ they were able to calculate a set of elastic constants for crystalline RDX. Another theoretical study by Sewell and Bennett ${ }^{14}$ reported a set of elastic constants for RDX at $276 \mathrm{~K}$ and 2.0 $\mathrm{GPa}$ of static pressure. In their calculations, Sewell and Bennett used isothermal-isobaric Monte Carlo methods, and employed different potential-energy surface parameters, also 
referenced to Sorescu et al. ${ }^{15}$ They also included extensive calculations of the crystal lattice parameters, anisotropic Young's and shear moduli, and Poisson ratios for RDX as a function of both temperature $(218-333 \mathrm{~K})$ and hydrostatic pressure (0-4 GPa), allowing for the calculation of elastic constants at a variety of temperatures and pressures.

This paper reports the elastic properties of the energetic material RDX, determined by Brillouin scattering. In the next section, a brief introduction to Brillouin scattering is given. All nine elastic constants of RDX are then presented and compared to the published results of earlier experimental and theoretical works. This is followed by a discussion of some specific elastic constants and linear compressibilities. Finally, a brief comparison to the stiffness constants of $\beta$-HMX is presented, and the role of elasticity with emphasis on shear deformation in recently developed models for detonation is briefly discussed.

\section{THEORY}

The principles of Brillouin scattering are well documented in the literature ${ }^{16,17}$ so only a brief description will be given. Acoustic phonons are the vibrations associated with translations of the center of mass in the unit cell. These phonons propagate through the lattice at the speed of sound. ${ }^{18}$ The phonon velocity is directly related to the strength of the force constants holding the mass in the unit cell in place. Brillouin spectroscopy probes acoustic phonons near the Brillouin-zone center, where the wavelengths of the phonons are on the order of hundreds of unit cells. This allows the material to be modeled as an elastic continuum, and is often referred to as the long-wavelength approximation. The inelastic scattering utilized in Brillouin spectroscopy is the result of diffraction from sound waves caused by thermal fluctuations in the lattice. In a classical sense, these lattice vibrations are compressional waves that can be viewed as a grating propagating through the lattice.

Each acoustic phonon in the lattice has one longitudinal component and two transverse components. The transverse components are at $90^{\circ}$ to the propagation direction, while the compressional component is along the propagation direction. Therefore, for any given phonon propagation direction, there are three possible modes that may be measured. However, experimental conditions may not allow for the direct measurement of all three using a single scattering geometry.

The change in frequency of the inelastically scattered light is small in Brillouin experiments, so the frequency of the incident light is approximately equal to the frequency of the scattered light giving

$$
\delta \omega_{a}= \pm \frac{v_{a} \omega_{i}}{c} \sqrt{n_{i}^{2}+n_{s}^{2}-2 n_{i} n_{s} \cos \theta},
$$

where $\delta \omega_{a}$ is the frequency shift of the inelastically scattered light, or simply the Brillouin shift, $v_{a}$ is the velocity of the acoustic mode in the material, $c$ is the speed of light in a vacuum, $\theta$ is the angle between the incident and scattering wave vectors, and $n_{i}$ and $n_{s}$ are the indices of refraction of the incident and scattered light, respectively. Equation (1) is the Brillouin-scattering equation for an anisotropic medium.
The Brillouin shift equation allows a calculation of the acoustic velocities which can be related to the elastic constants of the lattice. Starting with the equation of motion

$$
\rho \frac{\partial^{2} u_{i}}{\partial t^{2}}=C_{i j k l} \frac{\partial^{2} u_{j}}{\partial r_{j} \partial r_{k}}
$$

and then substituting plane-wave solutions of the form

$$
u_{i}=u_{i}^{0} \exp i\left(\mathbf{q} \cdot r-\omega_{a} t\right)
$$

yield the following equation:

$$
\left[C_{i j k l} \mathbf{q}_{j} \mathbf{q}_{k}-\rho \omega_{a}^{2} \delta_{i l}\right] u_{1}^{0}=0,
$$

where $C_{i j k l}$ are the elastic constants, $\mathbf{q}_{j}$ and $\mathbf{q}_{k}$ are the wave vectors of the acoustic waves, $\rho$ is the density of the material, $\omega_{a}$ is the frequency of the acoustic wave, and $\delta_{i l}$ is the Kronecker delta. Operating in the long-wavelength limit, Eq. (4) can be reduced to

$$
\left|C_{i j k l} \mathbf{q}_{j} \mathbf{q}_{k}-\rho v_{a}^{2} \delta_{i l}\right|=0 .
$$

Solving this Christoffel determinant gives the eigenvalues that are then used to calculate the elastic constants of the solid.

A calculation method initially utilized for elastic constant determination of cubic crystals and oriented polymer films has been modified for use in low-symmetry crystal systems. ${ }^{19-22}$ This method consists of an iterative, leastsquares minimization routine that solves for the elastic constants when the secular equations are experimentally overdetermined from many measurements of velocities in different directions. As the program goes through each iteration, the Christoffel determinant is solved for the eigenvalues, designated $\rho v_{a \text {,calc }}^{2}$, for each of the experimentally determined velocities using a set of trial elastic constants. The vector of differences, e, between these eigenvalues and the corresponding quantities, $\rho v_{a, \mathrm{obs}}^{2}$, from the experimentally observed velocities is then calculated. The square of the error vector

$$
\mathbf{e}^{T} \mathbf{e}=\sum_{j}\left|\rho \mathrm{v}_{a, \text { obs }}^{2}-\rho \mathrm{v}_{a, \text { calc }}^{2}\right|^{2}
$$

is then minimized by systematically varying all of the input elastic constants until a global minimum is reached. By utilizing this calculative technique, the accumulation of experimental error is avoided while still allowing for a complete determination of the stiffness tensor.

\section{EXPERIMENT}

Optical quality, untwinned, single crystals of RDX were grown evaporatively at $298 \mathrm{~K}$ from solutions of cyclohexanone saturated with de-ionized water. Crystals form in the orthorhombic space group $\mathrm{Pbca}$, with lattice constants $a$ $=13.182 \AA, b=11.574 \AA$, and $c=10.709 \AA$. There are eight molecules in the unit cell. The Pbca space group and lattice parameters were verified using single-crystal $\mathrm{x}$-ray diffraction and were equivalent within error to those reported by Choi and Prince. ${ }^{23}$ The crystals exhibited at least nine wellformed faces, eight of which were zonal to the $c$ crystallographic axis, $\{100\},\{010\},\{210\}$, and $\{\overline{2} 10\}$, with the remain- 
ing face, $\{001\}$, orthogonal to the eight zonal faces. In order to assign the faces, the interfacial angles between all zonal and capping faces were determined by optical goniometry. The Miller indices of the faces, which were subsequently corroborated by single-crystal $x$-ray diffraction, are necessary to precisely define the scattering angle $\theta$.

In addition to a well-defined scattering geometry, the refractive indices must be determined to solve Eq. (1). Because RDX is an orthorhombic crystal system, the axes of the optical indicatrix are coincident with the crystallographic axes. Therefore, it was only necessary to determine the nearnormal-incidence specular reflectivity along the principal directions for the (001), (010), and (100) faces in the crystal using a microspectroreflectometer described elsewhere. ${ }^{24}$ These reflectivities were measured at $514.5 \mathrm{~nm}$, the incident wavelength used in the Brillouin experiments reported here. The refractive indices for the relevant directions in the RDX crystal were calculated from the single wavelength reflectivities using Fresnel's Law under the approximation that $k \approx 0$ for a clear, colorless crystal at visible wavelengths.

Brillouin-scattering experiments were performed on three single crystals of RDX at $295 \mathrm{~K}$ and ambient pressure. A four-circle, Brillouin-scattering instrument ${ }^{25}$ was used that allows rotation of the incident light beam and crystal independently so that a large number of phonons can be accurately sampled. Frequency shifts were resolved using a Burleigh Fabry-Perot interferometer operated in the triple-pass configuration. Laser power was set at 200-300 mW, depending on the scattering geometry. To ensure that the Brillouin peaks of RDX were assigned to the correct spectral order, free spectral ranges (FSRs) of 1.079, 1.351, and $2.837 \mathrm{~cm}^{-1}$ were employed during this study. The FSRs were calibrated using the known shift of pure liquid benzene. ${ }^{26}$

Crystals were oriented in the incident light beam using back reflections from the specular input and output crystal faces. All four laboratory polarizations were utilized in each scattering geometry: Vertical-Vertical, Vertical-Horizontal, Horizontal-Vertical, and Horizontal-Horizontal, where the incident polarization setting is given first and the analyzer polarization is given last. Because of the orientation of the optical indicatrix, these polarizations were exactly along the crystallographic axes, thus negating the need to calculate intermediate angles for polarization. Each recorded spectrum was a summation of 300 interferometer scans.

\section{RESULTS}

An example Brillouin spectrum of RDX is shown in Fig. 1. The transverse slow, transverse fast, and longitudinal modes are all clearly present. The unusual occurrence of a longitudinal mode that is less intense than a transverse mode, observed for many of the Brillouin measurements for this material, was confirmed by utilizing Brillouin experiments with different FSRs. The minimized stiffness constants for this work are presented in the left column of Table I in standard Voigt notation. ${ }^{27}$ An orthorhombic crystal system requires a minimum of nine acoustic velocities to experimentally determine the stiffness tensor. For our reported values, an overdetermined set of 30 acoustic velocities measured in

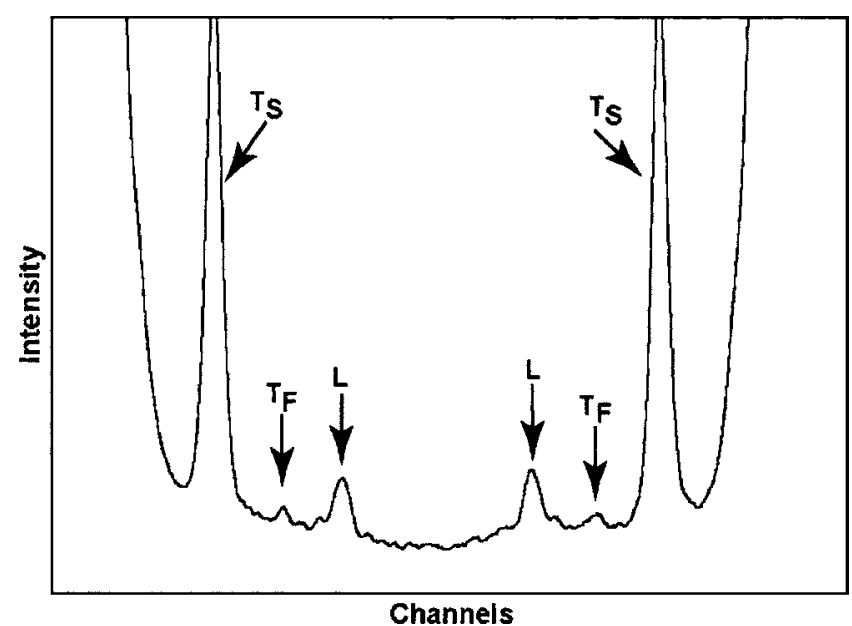

FIG. 1. Example Brillouin spectrum of RDX for the $(0.70710,0.70710$, $0.00000)$ phonon. The transverse slow $\left(T_{s}\right)$, transverse fast $\left(T_{f}\right)$, and longitudinal modes $(L)$ are labeled. The free spectral range is $1.369 \mathrm{~cm}^{-1}$. Polarization is laboratory Vertical-Vertical.

18 phonon directions was used in the least-squares minimization routine to solve for the stiffness constants. A density of $1806 \mathrm{~kg} / \mathrm{m}^{3}$ for RDX, calculated from the unit-cell parameters obtained by neutron diffraction, ${ }^{23}$ was used in the elastic constant calculation. Including an error of $\pm 0.5^{\circ}$ for angular crystal and polarization alignment and an error of $\pm 0.003 \mathrm{~cm}^{-1}$ for determination of the acoustic-mode energy shifts, the uncertainties in the reported elastic constants are on the order of $0.06 \mathrm{GPa}$. The principal minors of the stiffness tensor determinant were found to be positive, ensuring that the elastic constants represent a physically real system. ${ }^{28}$ The largest percent difference error between the calculated velocities and the experimental velocities was found to be $8.5 \%$ for the transverse slow mode of the $(0.70710$, $0.70710,0.00000)$ phonon. The arithmetic average percent difference error between the calculated velocities and the experimental velocities was $4.4 \%$ for the transverse slow, $2.3 \%$ for the transverse fast, and $1.2 \%$ for the longitudinal modes. These small differences in error indicate an excellent agreement between the measured and calculated acoustic velocities.

\section{DISCUSSION}

Table I presents the stiffness constant data from this work, the recent resonant ultrasonic study by Schwarz et al., ${ }^{8}$ the resonant ultrasonic study reported by Haussühl, ${ }^{7}$ the theoretical study published by Ye et al., ${ }^{9}$ and the theoretical study published by Sewell and Bennett. ${ }^{14}$ Haussühl chose the nonstandard $P c a b$ setting when solving the crystal structure of RDX, with lattice constants $a=11.618 \AA, b=13.199 \AA$, and $c=10.722 \AA$, and a calculated density of $1797 \mathrm{~kg} / \mathrm{m}^{3}$. The percent difference in density between this study and Haussühl's is approximately $0.5 \%$, which translates to a difference in the determined elastic constants on the order of $0.06 \mathrm{GPa}$, a value that matches the error in this study's elastic constant determination. However, this difference only negligibly contributes to the observed differences in the elastic constants. For the purpose of direct comparison, 
TABLE I. Comparisons between the various experimental and theoretical studies of the stiffness constants for RDX at ambient conditions. Haussühl's values have been transformed from a Pcab setting to the more common $P b c a$, for the purposes of direct comparison. Sewell and Bennett's values have been calculated using Young's and shear moduli and Poisson ratios. The stiffness constants are given by $C_{i j}$.

\begin{tabular}{cccccc}
\hline \hline Elastic constants (GPa) & This work & ${\text { Schwarz } \text { et al. }^{\mathrm{a}}}$ & Haussühl $^{\mathrm{b}}$ & Ye et al. & Sewell and Bennett $^{\mathrm{c}}$ \\
\hline$C_{11}$ & 36.67 & 25.60 & 25.02 & 28.91 & 26.90 \\
$C_{12}$ & 1.38 & 8.67 & 8.21 & 10.30 & 6.27 \\
$C_{13}$ & 1.67 & 5.72 & 5.81 & 6.53 & 5.68 \\
$C_{22}$ & 25.67 & 21.30 & 19.60 & 25.05 & 24.10 \\
$C_{23}$ & 9.17 & 6.40 & 5.90 & 7.59 & 6.32 \\
$C_{33}$ & 21.64 & 19.00 & 17.93 & 14.29 & 17.70 \\
$C_{44}$ & 11.99 & 5.38 & 5.17 & 5.14 & 8.40 \\
$C_{55}$ & 2.72 & 4.27 & 4.07 & 3.84 & 5.30 \\
$C_{66}$ & 7.68 & 7.27 & 6.91 & 4.24 & 7.60 \\
\hline \hline
\end{tabular}

\footnotetext{
Reference 8 .

${ }^{\mathrm{b}}$ Reference 7 .

${ }^{\mathrm{c}}$ Reference 9.

${ }^{\mathrm{d}}$ Reference 14.
}

Haussühl's published stiffness constants have been transformed into the Pbca setting and presented in Table I. Schwarz et al. reported a density of $1860 \mathrm{~kg} / \mathrm{m}^{3}$ for their RDX sample, and lattice constants consistent with literature values. The percent difference in density between this study and the study of Schwarz et al. is approximately 2.9\%, which produces a difference in the determined elastic constants on the order of $0.3 \mathrm{GPa}$, again a small contribution to the overall observed differences in the elastic constants. The theoretical study by Sewell and Bennett included a specific set of stiffness constants calculated at $2.0 \mathrm{GPa}$ of static pressure and a temperature of $276 \mathrm{~K}$. In addition, Sewell and Bennett ${ }^{14}$ calculated Young's and shear moduli and Poisson ratios as a function of temperature and pressure. Using these coefficients, it is possible to calculate a set of stiffness constants for RDX under ambient conditions. As such, we have used Sewell and Bennett's original coefficient data to calculate a set of elastic constants for RDX at $304 \mathrm{~K}$ and ambient pressure. The transformation of the space group of Haussühl's published stiffness constants and the calculation of an ambient set of stiffness constants from the coefficients published by Sewell and Bennett allow for a more complete and direct comparison between the different published works.

There are two general approximations for expressing the bulk modulus ${ }^{29}$ and the shear modulus. ${ }^{29}$ Using the Voigt approximation (uniform strain), the expression for the bulk modulus $\left(B_{v}\right)$ is

$$
B_{v}=\frac{1}{9} \sum_{i, j}^{3} C_{i j}
$$

and the shear modulus $\left(G_{v}\right)$ is

$$
\begin{aligned}
G_{v}= & \frac{1}{15}\left(C_{11}+C_{22}+C_{33}\right)-\frac{1}{15}\left(C_{12}+C_{23}+C_{13}\right) \\
& +\frac{1}{5}\left(C_{44}+C_{55}+C_{66}\right),
\end{aligned}
$$

where $C_{i j}$ are the elastic constants from the stiffness tensor, in matrix notation. Using the Reuss approximation (uniform stress), the expression for the bulk modulus $\left(B_{R}\right)$ is

$$
B_{R}=\left(\sum_{i, j}^{3} S_{i j}\right)^{-1}
$$

and the shear modulus $\left(G_{R}\right)$ is

$$
\begin{aligned}
G_{R}= & {\left[\frac{4}{15}\left(S_{11}+S_{22}+S_{33}\right)-\frac{4}{15}\left(S_{12}+S_{23}+S_{13}\right)\right.} \\
& \left.+\frac{1}{5}\left(S_{44}+S_{55}+S_{66}\right)\right]^{-1},
\end{aligned}
$$

where $S_{i j}$ are the compliance constants from the compliance

TABLE II. Comparisons between the various ambient-condition experimental and theoretical studies of the bulk $(B)$ and shear $(G)$ moduli for RDX.

\begin{tabular}{cccccc}
\hline \hline Moduli (GPa) & This work & Schwarz et al. $^{\mathrm{a}}$ & Haussühl $^{\mathrm{b}}$ & Ye et al. & Sewell and Bennett $^{\mathrm{d}}$ \\
\hline$B_{\text {Voigt }}$ & 12.05 & 11.94 & 11.38 & 13.01 & 11.68 \\
$B_{\text {Reuss }}$ & 11.92 & 11.67 & 11.05 & 11.51 & 11.34 \\
Avg. & 11.99 & 11.81 & 11.22 & 12.26 & 11.51 \\
& & & & & \\
$G_{\text {Voigt }}$ & 9.26 & 6.39 & 6.07 & 5.57 & 7.62 \\
$G_{\text {Reuss }}$ & 6.40 & 6.03 & 5.75 & 5.03 & 7.27 \\
Avg. & 7.83 & 6.21 & 5.91 & 5.30 & 7.45 \\
\hline \hline
\end{tabular}

\footnotetext{
${ }^{\mathrm{a}}$ Reference 8.

${ }^{\mathrm{b}}$ Reference 7 .

${ }^{\mathrm{c}}$ Reference 9 .

${ }^{\mathrm{d}}$ Reference 14.
} 


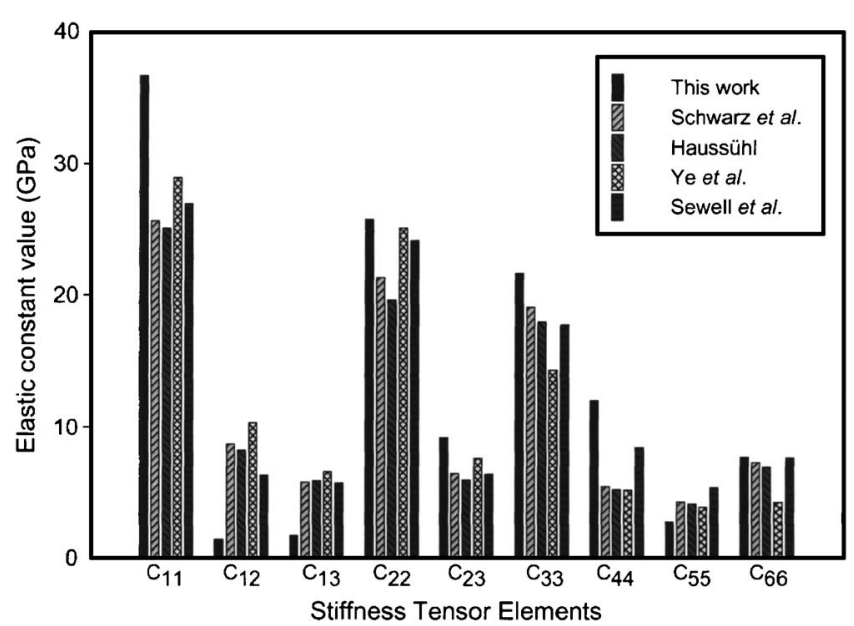

FIG. 2. Bar graph providing a visual comparison of the magnitude of the stiffness constants presented in Table I.

tensor, in matrix notation. Included in Table II are the bulk modulus and shear modulus using both the Voigt and Reuss approximations, as well as an arithmetic average (VoigtReuss-Hill) between the two approximations for the five sets of stiffness constants presented in Table I.

The Voigt-Reuss-Hill bulk moduli calculated from this work's experimental measurements show an excellent agreement with the results of Schwarz et al. and Ye et al., and a very good agreement with Haussühl and Sewell and Bennett. The Voigt-Reuss-Hill shear moduli agreement is not as good. While a very good agreement is seen with Sewell and Bennett, there is only a fair agreement with the remaining studies. The highest deviation from our values occurs with the Voigt approximation of the shear modulus, with a difference of $40.0 \%$ with respect to the value calculated from the data reported by Ye et al.

The agreement among the elements of the stiffness tensor is varied among the different researchers, as reported in Table I and graphically illustrated in Fig. 2. In all cases, the relative ordering of $C_{11}>C_{22}>C_{33}$ is observed. However, the values of $C_{11}, C_{22}$, and $C_{33}$ from this work are generally greater than those of the other researchers with the exception of $C_{22}$ which has a very close agreement to the value reported by Ye et al. For the other three diagonal elements of the stiffness tensor, this work, Ye et al., and Sewell and Bennett report that $C_{44}>C_{66}>C_{55}$, while Haussühl and Schwarz et al. report $C_{66}>C_{44}>C_{55}$. The numerical agreement between Schwarz et al., Haussühl, and Ye et al. is quite good for $C_{44}$ and $C_{55}$. This work reports $C_{44}$ to be much higher than the values reported by the other researchers. The lowest values for the off-diagonal elements, save that for $C_{23}$, are reported here. All the other researchers find $C_{12}>C_{13}$, while the opposite trend is found in this study. Although the absolute magnitudes of $C_{13}$ and $C_{23}$ vary somewhat among all previous works, the other researchers report similar relative internal values for $C_{13}$ and $C_{23}$. This work reports $C_{23}$ $\gg C_{13}$, an internal difference of $81.8 \%$. The values reported here for the stiffness constants differ somewhat from the previous experimental works by Schwarz et al. and Haussühl and the theoretical studies by Ye et al. and Sewell and Bennett, with most of the values from this work reported as
TABLE III. Comparisons between the longitudinal-acoustic velocities for $\beta$-HMX, along the (110), (011), and (010) directions. These velocities were determined using Brillouin scattering (Stevens et al.), impulsive stimulated light scattering (Zaug), and pulse-echo ultrasound (Dick et al.). The values for Zaug were calculated by Dick et al. in Ref. 31 .

\begin{tabular}{cccc}
\hline \hline \multirow{2}{*}{ Crystal direction } & \multicolumn{3}{c}{ Velocity (km/s) } \\
& Stevens et $_{\text {al. }}{ }^{\mathrm{a}}$ & Zaug & Dick et al. ${ }^{\mathrm{b}}$ \\
\hline$(110)$ & 2.92 & 3.30 & 3.76 \\
$(011)$ & 2.84 & 3.27 & 3.72 \\
$(010)$ & 2.75 & 3.76 & 3.17 \\
\hline
\end{tabular}

${ }^{\mathrm{a}}$ Reference 6.

${ }^{\mathrm{b}}$ Reference 31.

higher than those of the other four research publications.

Discrepancies between the theoretical studies and this experimental work are expected. In general, theoretical studies make use of potentials that often very accurately reproduce the unit-cell structure of a molecular system. However, this does not guarantee that the curvature of the potential surface would accurately predict the vibrational frequencies or elastic properties of a given material. ${ }^{30}$ As a consequence, theoretical reports on these types of properties often do not match well with the experiment. However, Ye et al. published comparisons to the work of Haussühl and reported a comparable agreement, although it appears that they neglected to transform the elastic constants reported by Haussühl into the Pbca space group. This error calls into question the validity of their assessment of a comparable agreement with Haussühl.

The more pertinent comparison, in terms of assessing the accuracy of the reported results, is between the experimental studies. Some deviation between the studies is expected given the nature and requirements of the two different experiments. Resonant ultrasonic measurements require large crystals that are often cut to obtain the necessary scattering geometries. Haussühl reported that his crystals were at least $1.0 \mathrm{~cm}$ in every dimension, while Schwarz et al. listed polished crystal dimensions of $7.326 \pm 0.033,4.216 \pm 0.032$, and $5.470 \pm 0.049 \mathrm{~mm}$. Large crystals are known to have higher numbers of defects that may be expected to affect the properties of the lattice. If enough defects are present in a crystal, variability in the measured values of the elastic constants and associated lack of precision may be expected. The RDX crystal habit published by Haussühl differs significantly from that observed for the crystals in this study. Haussühl's crystals displayed fewer unique faces and, more importantly, lacked any naturally occurring (001), (010), or (100) faces. Thus, the pure phonons associated with those crystallographic directions may not have been probed directly, leading to the possibility for error in some of the calculated elastic constants because such constants could not be obtained directly from the measured phonon velocities. Schwarz et al. utilized a crystal sample that had been cut from a much larger single crystal that was several years old. The cuboid crystal contained only the mutually perpendicular (100), (010), and (001) planes, which were cut using a low-speed diamond-impregnated wire saw using kerosene as a lubricant. This particular parallelepiped-shaped crystal would al- 

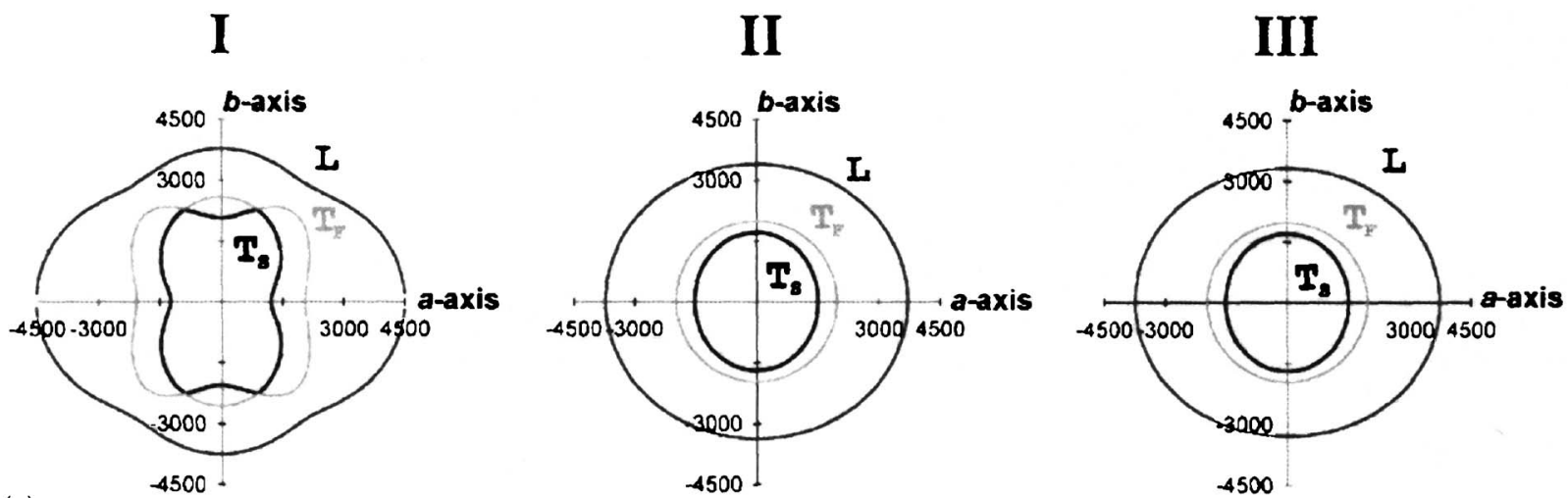

(a)
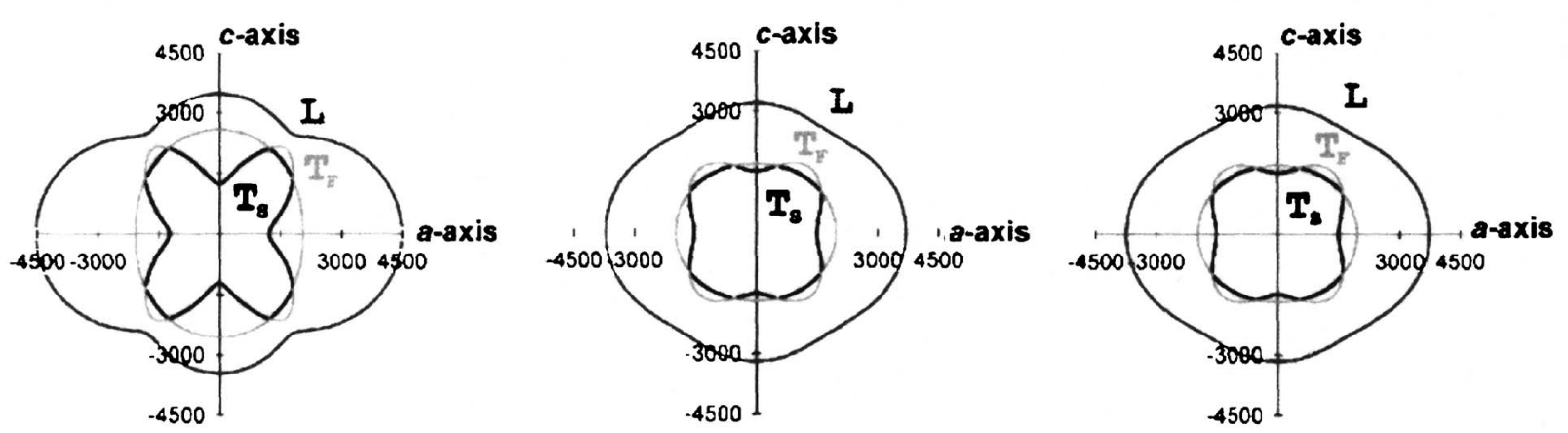

(b)
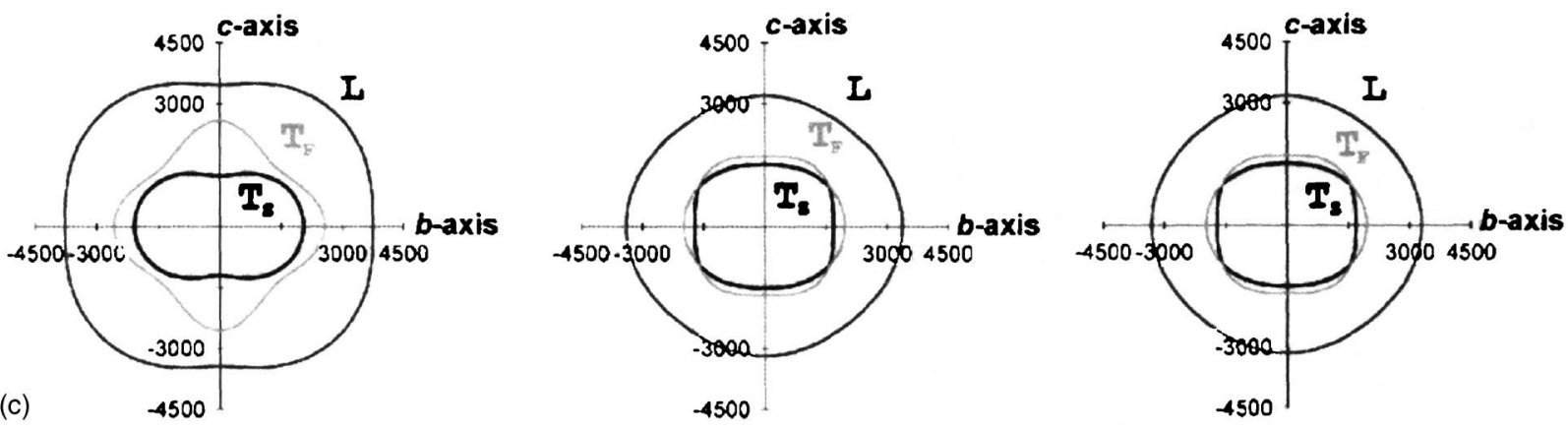

FIG. 3. Experimental sound velocity diagrams for RDX in the (a) $a b$ crystallographic plane, (b) $a c$ crystallographic plane, and (c) $b c$ crystallographic plane for this work (I), the resonant ultrasonic work of Schwarz (II), and the resonant ultrasonic work of Haussühl (III). The transverse slow $\left(T_{s}\right)$, transverse fast $\left(T_{f}\right)$, and longitudinal modes $(L)$ are labeled in each diagram. Units are in $\mathrm{m} / \mathrm{s}$.

low for the direct determination of acoustic-phonon velocities along the crystallographic axes. The lack of additional crystal faces meant that corroborating measurements on different acoustic phonons in the crystal could not be obtained. However, the crystal habits of our small, freshly grown crystals $\left(3.1 \times 2.0 \times 0.9 \mathrm{~mm}^{3}\right)$ exhibited a variety of faces that included the (100), (010), and (001) faces. This allowed for a larger number of unique acoustic phonons to be sampled and thus provided an extended self-consistency check of our stiffness tensor determination that was unavailable for the resonant ultrasound study of Schwarz et al. Furthermore, our use of naturally occurring crystal faces obviated the need for manufacturing crystal faces with a large amount of cutting or polishing which could significantly affect the elastic response, particularly for molecular crystals.

The Brillouin-scattering signal can only be observed for scattering volumes where the material is essentially pristine. Thus the resonant ultrasound and Brillouin experiments probably sample the material quite differently with the former more likely to encompass all the properties of the material while the latter can only be reliably obtained from quite small, nearly pristine regions of the lattice. These differences between the two experiments may, in part, account for the reported discrepancies in stiffness tensors, where the values obtained by Brillouin scattering may be either greater or lesser than those obtained by other methods.

Recently, Dick et al. made a comparison between the longitudinal-acoustic velocities along three directions in $\beta$-HMX measured in his work $^{31}$ with pulse-echo ultrasonic techniques and those calculated from the stiffness tensor determined with impulsive stimulated light scattering (ISLS) by Zaug. ${ }^{32}$ As listed in Table III, this comparison is extended to include the recently reported sound velocities determined by Brillouin scattering for $\beta$-HMX. ${ }^{6}$ The agreement between the separate determinations is marginal, with Stevens et al. containing the lowest values and Dick et al. reporting the 

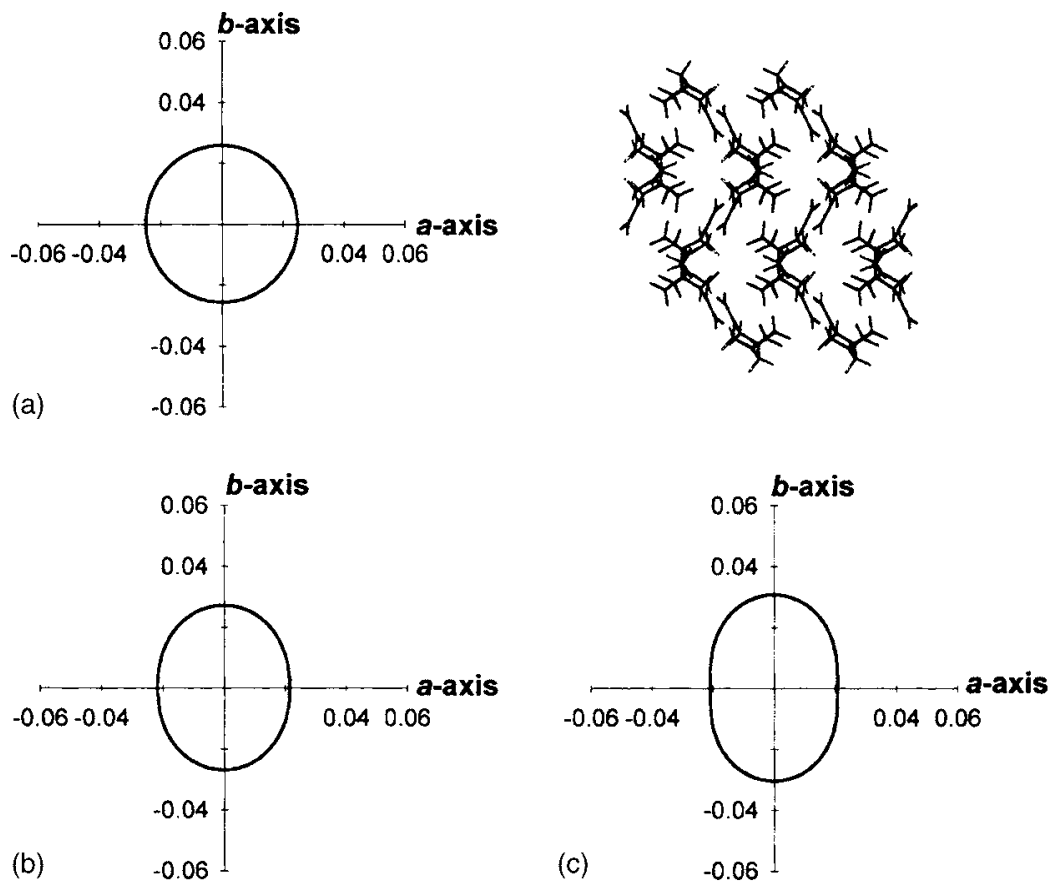

FIG. 4. Linear compressibility diagrams for RDX in the $a b$ crystallographic plane for (a) this work, (b) Schwarz et al., (c) Haussühl, (d) Ye et al., and (e) Sewell and Bennett. The projection of RDX molecules in the unit cell for this plane is shown in the upper-right corner. Units are in $\mathrm{GPa}^{-1}$.
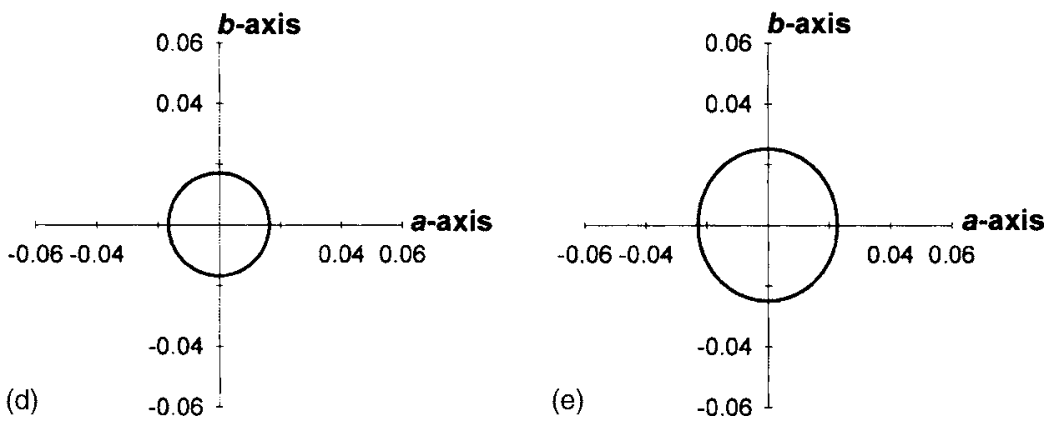

highest. While the immediate context pertains to $\beta$-HMX, the observations in comparison between the three methods can certainly be extended to other systems.

A survey of the current literature reveals that differences between measurement methods for elastic constant determinations is not a new phenomenon. However, certain trends can be observed in the magnitude of these differences, particularly in correlation to the symmetry of the lattice. For cases where the crystal system displays high symmetry, an excellent agreement between Brillouin and ultrasonic studies has been reported. Examples from the literature include $\mathrm{CD}_{4}$ (Ref. 33) and $\mathrm{SrFCl}^{34}$ For these high-symmetry systems, the equations of elasticity are simplified and, as a result, fewer measurements are required for a complete stiffness tensor determination. In addition, higher-symmetry systems also have fewer, off-diagonal tensor elements, which are known to contain the greatest sources of error. ${ }^{35}$ Due to the propensity of organic molecules to crystallize into low-symmetry space groups, elasticity tensor determinations for molecular crystals are limited. While this limitation prevents a complete description of observed discrepancies in acoustic velocities measured with complementary methods, a specific example for comparison is found in anthracene. Although this material has been well studied with both Brillouin ${ }^{36}$ and three independent ultrasonic studies, ${ }^{37-39}$ the published re- ports show large inconsistencies. These disagreements are not only between the elastic constants determined by Brillouin and ultrasound methods but also extend to the separate ultrasonic reports. While it could be stated that the Brillouin measurements of anthracene fall within the scatter of the results from the ultrasonic measurements, a quick analysis of each element of the stiffness tensor fails to highlight any definitive, consistent trends. Therefore, these disparities may only be partially explained by differences in the experimental approaches. Furthermore, the discrepancies in the reported stiffness tensors for anthracene only highlight the necessity of measuring a variety of acoustic phonons in multiple crystalline samples to obtain the most representative set of elastic constants for low-symmetry, organic molecular crystals. While symmetry is not the only deciding factor in differences in experimental methods, it can be used to explain some discrepancies in complementary techniques.

Because of this, correlation of the elastic constant values with other mechanical or structural properties of the crystal is desirable. RDX is an orthorhombic crystal system, so the $C_{11}, C_{22}$, and $C_{33}$ stiffness constants relate directly to the crystallographic $a, b$, and $c$ axes, respectively. Of these three constants, $C_{33}$ is found to be the weakest in this study. This is to be expected since RDX is reported to have a (001) cleavage plane. ${ }^{40}$ The existence of this cleavage plane was con- 

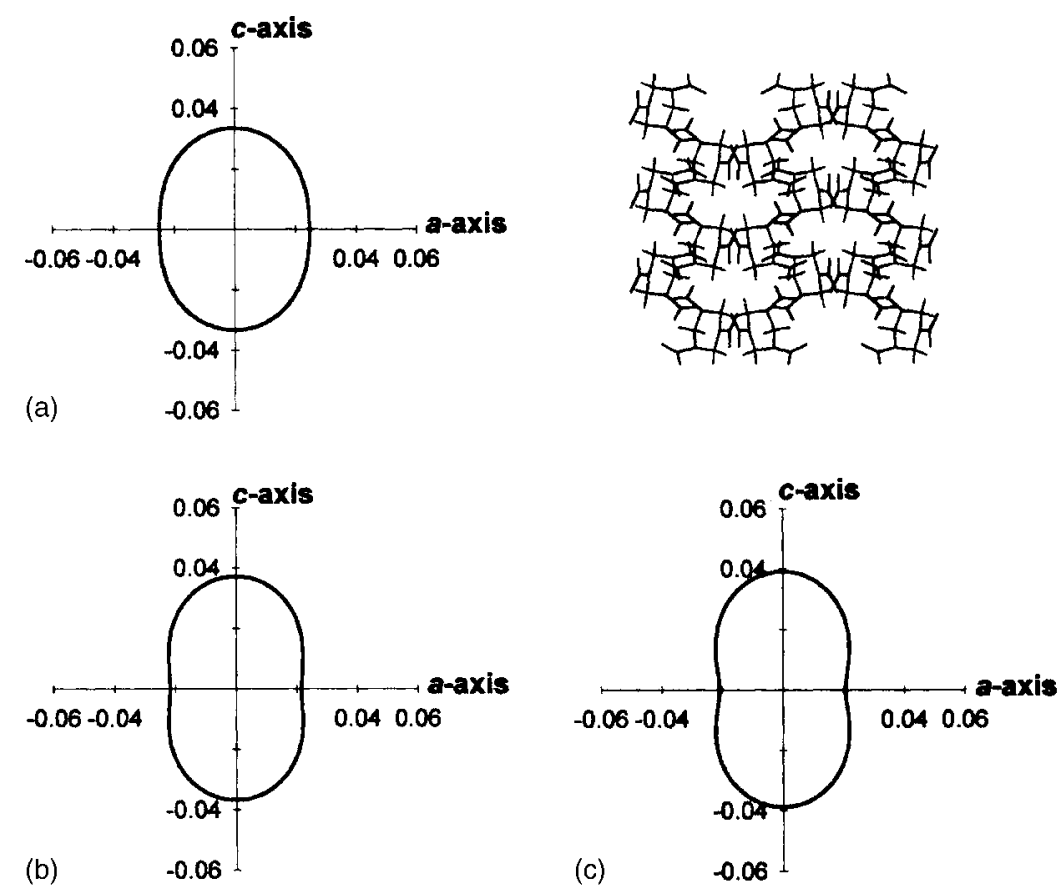

FIG. 5. Linear compressibility diagrams for RDX in the ac crystallographic plane for (a) this work, (b) Schwarz et al., (c) Haussühl, (d) Ye et al., and (e) Sewell and Bennett. The projection of RDX molecules in the unit cell for this plane is shown in the upper-right corner. Units are in $\mathrm{GPa}^{-1}$.
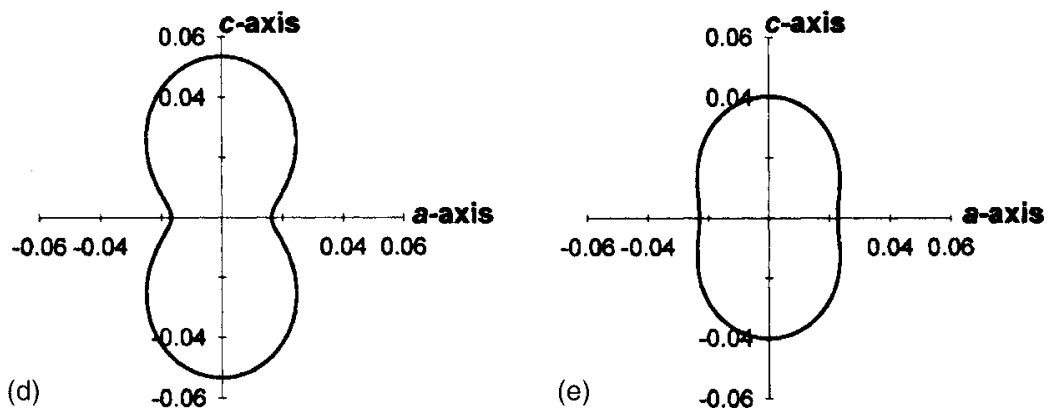

firmed and is good evidence of a relative weakness of lattice interactions along the crystallographic $c$ axis.

For the relative magnitudes of $C_{11}$ and $C_{22}$, the analysis of the crystal structure of RDX (Ref. 23) shows the largest number of close-contact (less than $3.0 \AA$ ), intermolecular interactions to be situated along the crystallographic $a$ axis. The increased number of interactions along the $a$ axis would stiffen the lattice in this direction which is consistent with the largest value of $C_{11}$. In addition, there are a significant number of interactions along the $b$ axis. Thus, we would expect to see a relatively large value for $C_{22}$, but less than the value for $C_{11}$. The lowest number of interactions is along the $c$ axis, which is further supported by the lowest value of $C_{33}$ and the existence of a cleavage plane. Both of the cited theoretical reports and the two resonant ultrasonic studies are in keeping with our observed trends for $C_{11}, C_{22}$, and $C_{33}$.

The off-diagonal, shear components of the stiffness tensor are $C_{12}, C_{13}$, and $C_{23}$. The constants $C_{12}$ and $C_{13}$ are found to be approximately equal in this study, but this is not reported elsewhere. $C_{12}$ and $C_{13}$ couple an applied normal stress component in the crystallographic $a$ direction with a uniaxial strain along the crystallographic $b$ and $c$ axes, respectively. The small values of these elastic constants would suggest that the crystal system is susceptible to shear along the crystallographic $b$ and $c$ axes, when a large force is ap- plied to the crystallographic $a$ axis. In addition, because $C_{22}$ and $C_{33}$ differ by only $16 \%$, similar magnitudes are expected for $C_{12}$ and $C_{13}$. $C_{23}$ has the largest value of the three shear components. It couples a uniaxial strain in the crystallographic $c$ direction to an applied normal stress component in the crystallographic $b$ direction. The larger value of $C_{23}$ mirrors the observation that the values of $C_{22}$ and $C_{33}$ are similar in magnitude. This means that there is no large discrepancy in the strength of the intermolecular forces along either of these two directions. This balance of forces along the separate crystallographic axes could cause an increased resistance to shear, relative to the other off-diagonal tensor elements.

\section{Cauchy relations}

Cauchy relations ${ }^{41}$ may be used to assess the anisotropy of the lattice potential. For structures that are centrosymmetric and strictly follow a central potential, the elastic constants should be totally symmetric in their four suffixes. For an orthorhombic space group, these relations are $C_{23}$ $=C_{44}, C_{13}=C_{55}$, and $C_{12}=C_{66}$. The further symmetry provided by the Cauchy relations reduces the maximum number of independent elastic constants from 21 to 15 for the totally general case. In crystal structures for which the Cauchy relations might be valid, the extent to which they are fulfilled 

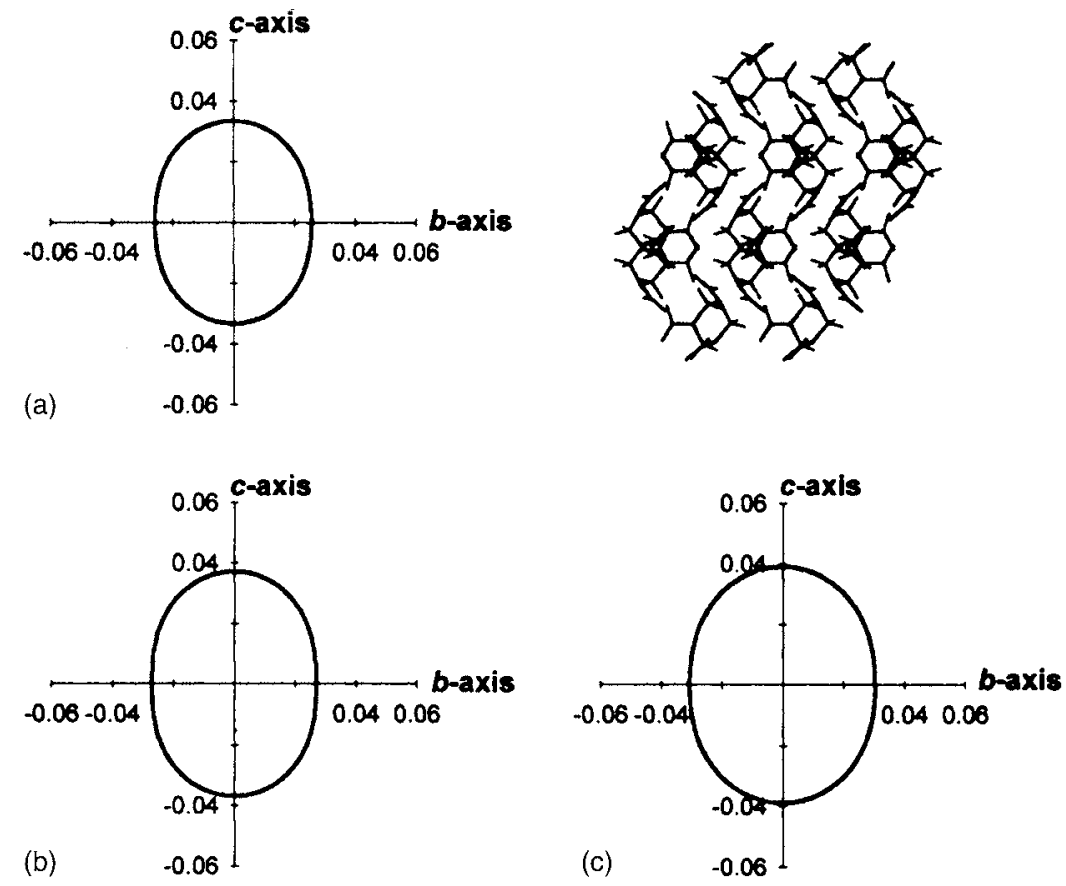

FIG. 6. Linear compressibility diagrams for RDX in the $b c$ crystallographic plane for (a) this work, (b) Schwarz et al., (c) Haussühl, (d) Ye et al., and (e) Sewell and Bennett. The projection of RDX molecules in the unit cell for this plane is shown in the upper-right corner. Units are in $\mathrm{GPa}^{-1}$.
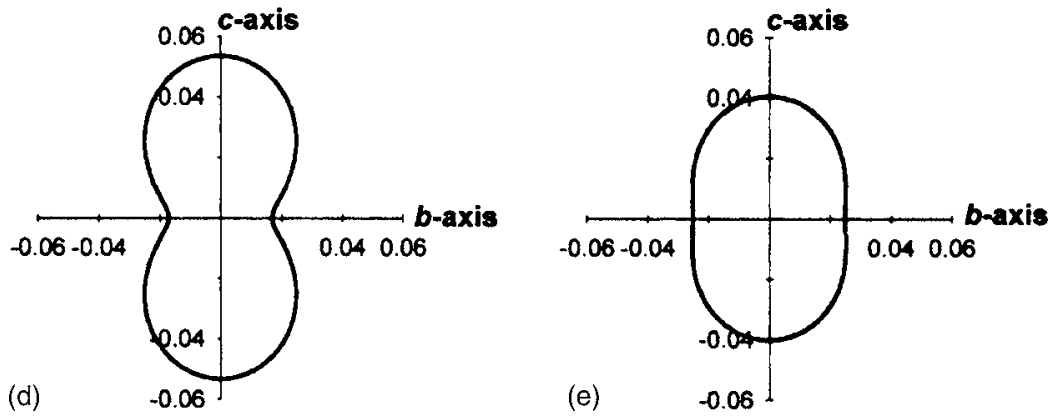

louin and resonant ultrasonic results show limited similari-

is often used to assess the validity of assuming a centralforce field. For the RDX stiffness constants reported in this work, $C_{23}$ and $C_{44}$ differ by $24 \%$ and $C_{13}$ and $C_{55}$ by $39 \%$. Finally, with $C_{12}$ and $C_{66}$ differing by $82 \%$, it can be said that all of these constants differ by a substantial amount, indicating that the Cauchy relations are far from applicable for RDX. This is a clear indication that many-body forces are required to accurately predict the properties of RDX in the solid state.

\section{Sound velocity diagrams}

Figure 3 gives the sound velocity diagrams of RDX for the crystallographic $a b, a c$, and $b c$ planes. The transverse slow, transverse fast, and longitudinal modes are labeled. Each diagram is a two-dimensional slice that represents the velocity at which a sound wave propagates through the lattice in a given plane. The elastic constants published by Haussühl, transformed to the $P b c a$ space group, and the elastic constants published by Schwarz et al. were used to determine sound velocity diagrams so that comparisons with the resonant ultrasonic measurements can be made.

The sound velocity diagrams of Schwarz et al. and Haussühl are virtually indistinguishable, displaying only the slightest variations in velocity. This is due to the good agreement of their stiffness tensors. Comparison between the Bril- ties. The $a b$ plane shows that surface shapes and velocities differ substantially between the two types of experimental studies. The $b c$ plane shows similar velocities but different surface shapes. The $a c$ plane shows similar shapes, but differing velocities, between this work and the resonant ultrasonic studies, thereby representing the closest match of the three orthogonal planes. The differences in shape and velocity in the diagrams are directly tied to the reported stiffness tensors and the material's measured density.

\section{Linear compressibility}

Linear compressibility is a measure of how the lattice responds to a hydrostatic stress. The larger the value, the more compliant the lattice is along a given stress direction. The compressibility is a function of direction and can provide insight into the anisotropy of the strength of intermolecular interactions. A direction in which the intermolecular interactions are stronger would reflect a lower compressibility in that direction. The linear compressibility is calculated from

$$
\beta=\sum_{i} \sum_{j} S_{i j k l} \mathbf{q}_{i} \mathbf{q}_{j}
$$

where $S_{i j k l}$ represents the compliance tensor. A polar plot of Eq. (11) provides association of the entire compliance tensor 
and compressibility which portrays the tensor property rather than using single elements of the tensor that have a greater projection along a given direction. Figures 4-6 show the linear compressibilities of RDX in each of the three crystallographic planes using the compliance tensors calculated from all of the elastic constant data listed in Table I. The projection of RDX in the unit cell in each of the three crystallographic planes is also included in the upper-right-hand corner for each figure, as a spatial reference.

The compressibility diagrams provide information on how easily the lattice deforms under a given hydrostatic stress. A plot that is circular indicates isotropic behavior under compression while plots that are elliptical indicate an anisotropy in the compressibility. This work, Ye et al., and Sewell and Bennett display a circular shape in the plot for the $a b$ crystallographic plane, indicating nearly isotropic behavior. The elliptical shapes displayed by Schwarz et al. and Haussühl indicate a higher degree of compressibility along the $b$ axis. Ye et al. displays the lowest compressibility values for this crystallographic plane, indicated in Fig. 4(d) by the smaller radius of the circular plot. For the $a c$ crystallographic plane, shown in Fig. 5, the oval shape of this work indicates a small deviation from isotropic compressibility in this plane. The more distorted oval shapes seen in (b)-(d) indicate a larger deviation from isotropic compressibility. Finally, for the $b c$ plane shown in Fig. 6, the presence of a slight deviation from a circle for this work shows that RDX has nearly isotropic behavior in this plane. The more elliptical shapes of Schwarz et al., Haussühl, and Sewell and Bennett show a higher degree of compressibility along the $c$ axis, as compared to the $b$ axis. This difference is also clearly illustrated in the peanut-shaped plot of the data of Ye et al.

The nearly isotropic compressibility calculated from the measured stiffness constants reported here is in good agreement with recent experimental and theoretical works. Olinger et al. $^{42}$ found that under hydrostatic compression up to approximately $1 \mathrm{GPa}$, all three lattice dimensions of RDX decreased by nearly equal percentages. From 1 to $4 \mathrm{GPa}$, some anisotropy is seen, with the percent change in the length of the crystallographic $c$ axis being the greatest. A theoretical calculation by Sewell ${ }^{43}$ was found to be in excellent agreement with those experimental findings. The uniform compression of the unit cell in all three dimensions under moderate applied hydrostatic pressure indicates approximate isotropic compressibility in crystals of RDX in this pressure region. Plotting the linear compressibilities of the other researchers' work reveals significant anisotropy in some of the three crystallographic planes, particularly the $a c$ and $b c$ planes. This indicates a significant deviation from the reported behavior based upon hydrostatic compression studies published by others. The discrepancy between the resonant ultrasonic works and the hydrostatic compression studies by Olinger et al. suggests that the elastic constants calculated from the Brillouin measurements reported here better reproduce the overall elastic response of RDX in the elastic regime.
TABLE IV. Comparisons between common stiffness constants from experimental studies on RDX and $\beta$-HMX in our laboratory. $\beta$-HMX crystallizes in the monoclinic space group $P_{2_{1} / n}$.

\begin{tabular}{ccc}
\hline \hline $\begin{array}{c}\text { Elastic constants } \\
(\mathrm{GPa})\end{array}$ & $\begin{array}{c}\text { RDX } \\
(\text { This work })\end{array}$ & $\begin{array}{c}\beta \text {-HMX } \\
\left(\text { Stevens } \text { et al. }{ }^{\mathrm{a}}\right)\end{array}$ \\
\hline$C_{11}$ & 36.67 & 18.41 \\
$C_{12}$ & 1.38 & 6.37 \\
$C_{13}$ & 1.67 & 10.50 \\
$C_{22}$ & 25.67 & 14.41 \\
$C_{23}$ & 9.17 & 6.42 \\
$C_{33}$ & 21.64 & 12.44 \\
$C_{44}$ & 11.99 & 4.77 \\
$C_{55}$ & 2.72 & 4.77 \\
$C_{66}$ & 7.68 & 4.46 \\
\hline \hline
\end{tabular}

${ }^{\mathrm{a}}$ Reference 6 .

\section{Comparison of RDX to $\beta$-HMX}

Table IV lists the stiffness constants common between RDX and $\beta$-HMX using the data reported in this work and previously by our laboratory. ${ }^{6}$ Since $\beta$-HMX is a monoclinic system, its stiffness tensor is comprised of 13 independent elastic constants. Thus, a direct comparison of all stiffness constants between the two materials is not possible. However, some observations may be made concerning some of the more prominent stiffness constants. The ordering of $C_{11}$ $>C_{22}>C_{33}$ is common to both materials, illustrating they have the strongest interactions along the $a$ crystallographic axis. Additionally, the lowest elastic constant for the series $C_{11}, C_{22}$, and $C_{33}$ in both RDX and $\beta$-HMX is reflective of their respective cleavage planes. In general, the listed values for RDX are much higher than those of $\beta$-HMX, particularly for the $C_{11}$ (99\% higher), $C_{22}\left(78 \%\right.$ higher), and $C_{33}(74 \%$ higher) constants. The notable exceptions are for $C_{12}$ and $C_{13}$, which are $78 \%$ and $84 \%$ lower than the same constants in $\beta$-HMX. These low values suggest that RDX crystals will be more susceptible to specific shear deformations along the $b$ and $c$ crystallographic axes.

Table $\mathrm{V}$ lists the bulk and shear moduli comparisons between RDX and $\beta$-HMX. The Voigt-Reuss-Hill bulk moduli differ by $17.4 \%$, while the Voigt-Reuss-Hill shear moduli differ by $52.7 \%$. The larger shear modulus value for RDX indicates that the solid is overall less susceptible to

TABLE V. Comparisons between the bulk $(B)$ and shear $(G)$ moduli obtained from experimental studies on RDX and $\beta$-HMX in our laboratory.

\begin{tabular}{ccc}
\hline \hline $\begin{array}{c}\text { Moduli } \\
(\mathrm{GPa})\end{array}$ & $\begin{array}{c}\text { RDX } \\
(\text { This work })\end{array}$ & $\begin{array}{c}\beta \text {-HMX } \\
\left(\text { Stevens } \text { et al. }{ }^{\mathrm{a}}\right)\end{array}$ \\
\hline$B_{\text {Voigt }}$ & 12.05 & 10.20 \\
$B_{\text {Reuss }}$ & 11.92 & 9.60 \\
Avg. & 11.99 & 9.90 \\
& & \\
$G_{\text {Voigt }}$ & 9.26 & 4.30 \\
$G_{\text {Reuss }}$ & 6.40 & 3.10 \\
Avg. & 7.83 & 3.70 \\
\hline \hline
\end{tabular}

${ }^{a}$ Reference 6 
shear forces than $\beta$-HMX. However, the low values of $C_{12}$ and $C_{13}$ indicate that RDX is highly susceptible to shear only along those specific directions.

\section{Relevance to detonation}

How these elastic constants directly relate to detonation is still cause for speculation. It is possible that the stiffer the lattice, the less sensitive it is to detonation initiation from a mechanical shock. From the elastic constants published by our laboratory for RDX and $\beta$-HMX, we have found that RDX has the stiffer lattice of the two materials. A recent report found HMX to be more sensitive to shock-initiated detonation than $\mathrm{RDX},{ }^{44}$ although no mention of lattice directional sensitivity was made. In addition the elastic constants of PETN (Ref. 4) are generally smaller than those of RDX, and slightly smaller than those of $\beta$-HMX. Of these three energetic materials, PETN is found to be the most sensitive to shock initiation. Furthermore, the association of lattice stiffness and orientation-dependent detonation sensitivity is observed for PETN, whose stiffest elastic constant is $C_{11}$ with the lowest detonation sensitivity along the (100) crystallographic direction. ${ }^{5}$ If this is found to be a general trend among secondary nitramine explosives, then RDX should be most sensitive to detonation along the crystallographic $c$ axis and least sensitive along the $a$ axis. The small values of $C_{12}$ and $C_{13}$ indicate that the crystal is susceptible to shear that could make the crystal more sensitive to certain oriented shocks, a necessary precursor for the Gilman ${ }^{45}$ and LutyOrdon-Eckhardt models ${ }^{46,47}$ of detonation initiation.

\section{CONCLUSIONS}

The stiffness constants for RDX have been successfully determined using Brillouin scattering. These constants were found to exhibit trends similar to those seen by other researchers, with values lying generally greater than those reported in other work. These measurements indicate that RDX is stiffest along the crystallographic $a$ axis, followed by $b$ and then $c$ axes. However, when considering the entire stiffness tensor, the linear compressibility diagrams indicate nearly isotropic compressibility under moderate applied pressures. This is corroborated by the hydrostatic compression experiments published by other researchers.

\section{ACKNOWLEDGMENTS}

Support of this research by the U.S. Office of Naval Research under Grant No. N000149810736 and by Pfizer, Inc. is gratefully acknowledged.

${ }^{1}$ G. M. J. Schmidt, Pure Appl. Chem. 27, 647 (1971).

${ }^{2}$ M. D. Cohen and G. M. J. Schmidt, J. Chem. Soc. 1964, 1996; M. D. Cohen, G. M. J. Schmidt, and F. I. Sonntag, ibid. 1964, 2000.

${ }^{3}$ H. H. Cady and L. C. Smith, Los Alamos Technical Report No. LAMS2652 (Los Alamos National Laboratory, Los Alamos, NM, 1962).

${ }^{4}$ J. M. Winey and Y. M. Gupta, J. Appl. Phys. 90, 1669 (2001).

${ }^{5}$ C. S. Yoo, N. C. Holmes, P. C. Souers, C. J. Wu, F. H. Ree, and J. J. Dick, J. Appl. Phys. 88, 70 (2000).

${ }^{6}$ L. L. Stevens and C. J. Eckhardt, J. Chem. Phys. 122, 174701 (2005).

${ }^{7}$ S. Haussühl, Z. Kristallogr. 216, 339 (2001).
${ }^{8}$ R. B. Schwarz, D. E. Hooks, J. J. Dick, J. I. Archuleta, and A. R. Martinez, J. Appl. Phys. 98, 056106 (2005).

${ }^{9}$ S. Ye, K. Tonokura, and M. Koshi, Kayaku Gakkaishi 63, 104 (2002).

${ }^{10}$ J. D. Gale, Philos. Mag. B 73, 3 (1996).

${ }^{11}$ D. C. Sorescu, B. M. Rice, and D. L. Thompson, J. Phys. Chem. B 104, 8406 (2000).

${ }^{12}$ E. P. Wallis and D. L. Thompson, J. Chem. Phys. 99, 2661 (1993).

${ }^{13}$ C. C. Chambers and D. L. Thompson, J. Phys. Chem. 99, 15881 (1995).

${ }^{14}$ T. Sewell and C. Bennett, J. Appl. Phys. 88, 88 (2000).

${ }^{15}$ D. C. Sorescu, B. M. Rice, and D. L. Thompson, J. Phys. Chem. B 101, 798 (1997).

${ }^{16}$ H. Z. Cummins and P. E. Schoen, in Laser Handbook, edited by F. T. Arecchi and E. O. Schulz-Dubois (North-Holland, Amsterdam, 1972), Chap. E1.

${ }^{17}$ I. L. Fabelinskii, Molecular Scattering of Light (Plenum, New York, 1968).

${ }^{18}$ D. F. Nelson, Electric, Optic, \& Acoustic Interactions in Dielectrics (Wiley, New York, 1979).

${ }^{19}$ S. F. Ahmad, H. Kiefte, M. J. Clouter, and M. D. Whitmore, Phys. Rev. B 26, 4239 (1982)

${ }^{20}$ D. B. Cavanaugh and C. H. Wang, J. Chem. Phys. 52, 5998 (1981).

${ }^{21}$ D. B. Cavanaugh and C. H. Wang, J. Chem. Phys. 53, 2793 (1982).

${ }^{22}$ K. H. Brose and C. J. Eckhardt, Chem. Phys. Lett. 125, 235 (1986).

${ }^{23}$ C. S. Choi and E. Prince, Acta Crystallogr., Sect. B: Struct. Crystallogr. Cryst. Chem. 28, 2857 (1972). A more recent x-ray determination by Singh and Kaur [Curr. Sci. 78, 1184 (2000)] was found to be in substantial agreement with the previous neutron-diffraction study by Choi and Prince. The structure by Singh and Kaur was solved in the nonstandard $P c a b$ setting and has a nearly identical calculated density to that of Choi and Prince. Thus no changes in any calculated values in this publication would be necessary and only the labeling of specific elements of the stiffness tensor would be affected by the change in space-group setting.

${ }^{24}$ C. J. Eckhardt and R. R. Pennelly, Chem. Phys. Lett. 9, 572 (1971).

${ }^{25}$ R. C. Dye, J. Sartwell, and C. J. Eckhardt, Rev. Sci. Instrum. 60, 2610 (1989).

${ }^{26}$ H. Z. Cummins and R. W. Gammon, J. Chem. Phys. 44, 2785 (1966).

${ }^{27}$ J. F. Nye, Physical Properties of Crystals (University Press, London, 1957).

${ }^{28}$ M. Born and K. Huang, Dynamical Theory of Lattices (Oxford University Press, Oxford, 1954).

${ }^{29}$ E. Schreiber, O. L. Anderson, and N. Soga, Elastic Constants and Their Measurement (McGraw-Hill, New York, 1973). Error present in Eq. (2.22) on page 30.

${ }^{30}$ G. M. Day, S. L. Price, and M. Leslie, Cryst. Growth Des. 1, 13 (2001).

${ }^{31}$ J. J. Dick, D. E. Hooks, R. Menikoff, and A. R. Martinez, J. Appl. Phys. 96, 374 (2004).

${ }^{32}$ J. M. Zaug, Proceedings of the 11th Detonation Symposium, Snowmass, CO, 1998, p. 498.

${ }^{33}$ S. V. Marx and R. O. Simmons, J. Chem. Phys. 81, 944 (1984).

${ }^{34}$ M. Fisher, A. Polian, and M. Sieskind, J. Phys.: Condens. Matter 6, 10407 (1994).

${ }^{35}$ K. H. Brose and C. J. Eckhardt, Chem. Phys. Lett. 125, 235 (1986).

${ }^{36}$ R. C. Dye and C. J. Eckhardt, J. Chem. Phys. 90, 2090 (1989).

${ }^{37}$ G. K. Afanaseva, K. S. Aleksandrov, and A. J. Kitaigorodskii, Phys. Status Solidi 24, 61 (1967)

${ }^{38}$ T. Danno and H. Inokuchi, Bull. Chem. Soc. Jpn. 41, 1783 (1968).

${ }^{39}$ H. B. Huntington, S. G. Gangoli, and J. L. Mills, J. Chem. Phys. 50, 3844 (1969).

${ }^{40}$ Z. Galdecki, P. Grochulski, Z. Wawrzak, and J. Stepien-Damm, Phys. Status Solidi A 83, K113 (1984).

${ }^{41}$ M. J. P. Musgrave, Crystal Acoustics: An Introduction to the Study of Elastic Waves and Vibrations in Crystals (Holden-Day, San Francisco, 1970).

${ }^{42}$ B. Olinger, B. Roof, and H. Cady, Symposium International Sur le Comportement Des Milieux Denses Sous Hautes Pressions Dynamiques, Paris, France, 1978 (Commissariat a l'Energie Atomique Centre d'Etudes de Vajours, Sevran, France 1978), p. 3.

${ }^{43}$ T. D. Sewell, J. Appl. Phys. 83, 4142 (1998).

${ }^{44}$ H.-H. Licht, Propellants, Explos., Pyrotech. 25, 126 (2000).

${ }^{45}$ J. J. Gilman, Philos. Mag. B 71, 1057 (1995).

${ }^{46}$ T. Luty and C. J. Eckhardt, J. Am. Chem. Soc. 117, 2441 (1995).

${ }^{47}$ T. Luty, P. Ordon, and C. J. Eckhardt, J. Chem. Phys. 117, 1 (2002). 